\title{
mr-PosEBR - A novel positive tone resist for high resolution electron beam lithography and 3D surface patterning
}

\author{
Stefan Pfirrmann ${ }^{\mathrm{a}^{*}}$, Robert Kirchner ${ }^{\mathrm{b}}$, Olga Lohse ${ }^{\mathrm{c}}$, Vitaliy A. Guzenko ${ }^{\mathrm{b}}$, Anja Voigt ${ }^{\mathrm{a}}$, Irina Harder ${ }^{\mathrm{c}}$, \\ Anett Kolander ${ }^{\mathrm{a}}$, Helmut Schift ${ }^{\mathrm{b}}$, Gabi Grützner ${ }^{\mathrm{a}}$ \\ ${ }^{a}$ micro resist technology GmbH, Köpenicker Str. 325, 12555 Berlin, Germany \\ ${ }^{\mathrm{b}}$ Paul Scherrer Institut, 5232 Villigen PSI, Switzerland \\ ${ }^{c}$ Max Planck Institute for the Science of Light, Staudtstr. 7/B2, 91058 Erlangen, Germany
}

\begin{abstract}
In this contribution, we present the results of a systematic material variation for the development of a resist material for high resolution positive tone electron beam lithography (EBL). Several acrylic copolymer materials with different compositions, that is varying mass fractions of the comonomers and different molecular weights, were synthesized and as resist solutions - evaluated in terms of EBL performance at acceleration voltages of $30 \mathrm{kV}$ and $100 \mathrm{kV}$. The resist material exhibiting the best combination of the desired properties, named mr-PosEBR, is two times more sensitive than PMMA 495k and performs comparably to the known high resolution resist ZEP520A at $30 \mathrm{kV}$. For example, a grating pattern with $29 \mathrm{~nm}$ wide lines with a period of $100 \mathrm{~nm}$ could be lithographically generated in films of mr-PosEBR with an area dose of $100 \mu \mathrm{C} / \mathrm{cm}^{2}$. In terms of resolution, single lines of only $35 \mathrm{~nm}$ width could be fabricated via metal liftoff. Furthermore, the dry etch stability of mr-PosEBR in a $\mathrm{CF}_{4} / \mathrm{SF}_{6}$ process is similar to the one of ZEP520A. Consequently, via dry etching nano patterns in mr-PosEBR could be smoothly transferred into the underlying $\mathrm{Si}$ substrate with high fidelity. Moreover, mr-PosEBR was evaluated as electron beam grayscale patterning and reflow resist. It was shown that the resist exhibits a good grayscale and reflow performance very similar to PMMA 120k and ZEP520A. Via these well controllable processes the generation of a wide variety of features and applications is possible.
\end{abstract}

Keywords: electron beam lithography, positive tone, grayscale, reflow, resist material, high resolution, nanopatterning

\section{INTRODUCTION}

Electron beam lithography (EBL) represents one of the most important nanofabrication techniques: On the one hand it allows for the reliable direct writing of arbitrary two-dimensional patterns with lateral resolution down to the nanometer scale. On the other hand it enables high volume nanoscale patterning technologies such as optical lithography as well as nanoimprint lithography through the formation of masks and templates with high resolution. ${ }^{1}$ One disadvantage of EBL is that it used to be relatively slow for high throughput volume manufacturing in many cases. Therefore, it has been put significant effort into the improvement of the performance of EBL tools in recent years which has led to the development of multi-beam and hybrid technologies. ${ }^{2}$ Besides the technical advancements of EBL systems the resist materials used are vital for high quality and high throughput EBL. For an ideal EBL resist material it is desirable to have high sensitivity and high contrast but also high resolution and high etch stability for an efficient pattern transfer into the substrate. ${ }^{3}$ One of the most widely used positive tone, high resolution EBL organic polymer resists is high molecular weight poly(methyl methacrylate) (PMMA, Figure 1), mainly due to its excellent achievable resolution. ${ }^{4}$

*s.pfirrmann@microresist.de; microresist.com

Advances in Patterning Materials and Processes XXXIII, edited by Christoph K. Hohle, Todd R. Younkin, Proc. of SPIE Vol. 9779, 977925 - @ 2016 SPIE · CCC code: 0277-786X/16/\$18 · doi: 10.1117/12.2219165 
<smiles>COC(=O)C(C)(C(C)(C)C)C(C)(C)C</smiles>

poly(methyl methacrylate)

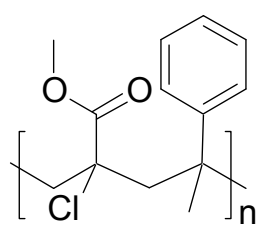

ZEP520A<smiles>C=C(Cl)C(=O)OC</smiles>

methyl $\alpha$-chloroacrylate

Figure 1. Schematic representation of the chemical structures of the polymers poly(methyl methacrylate) (PMMA), ZEP520A, and the monomer methyl $\alpha$-chloroacrylate.

Although PMMA (e.g. PMMA 495k, Microchem Corp.) provides high resolution at little cost, its performance is limited in terms of sensitivity and dry etch resistance. ${ }^{5-7}$ The low sensitivity of PMMA resist results in higher exposure doses and hence in longer exposure times, which is unfavorable for high throughput and large area applications. The low dry etch resistance of PMMA is also detrimental for an effective resist pattern transfer into the underlying substrate by dry etch processes. Another well-established positive tone EBL resist is ZEP520A (Zeon Corp.), a 1:1 copolymer of methyl $\alpha$-chloroacrylate (MCA) and $\alpha$-methylstyrene, that has been on the market for more than 25 years (Figure 1). ${ }^{8}$ Compared to PMMA, ZEP520A is more sensitive and especially more etch resistant, but at the same time it exhibits a similar resolution as PMMA. ${ }^{9}$

The higher sensitivity of the ZEP520A in comparison to PMMA is assigned to the presence of the MCA comonomer. The electron withdrawing effect of the chlorine atom on the quaternary carbon weakens the main chain stability of the polymer. Consequently, MCA containing polymers are degraded more easily by radiation (e. g. electron beams) via main chain scission than PMMA. ${ }^{5}$ At the same time, the homopolymer poly(methyl $\alpha$-chloroacrylate) not only shows main chain scission following irradiation but also tends to significant crosslinking, especially at higher radiation doses. This is unfavorable for a positive tone resist as it leads to decreased electron beam sensitivity. It was shown previously that it is possible to reduce the tendency towards crosslinking for copolymers containing MCA by adjusting the MCA content while keeping the tendency towards main chain scission high. ${ }^{5,10}$ Furthermore, the choice for $\alpha$-methylstyrene as the second comonomer in ZEP520A is presumably motivated by the known relatively high dry etch durability of aromatic compounds. ${ }^{7}$

Due to its good lithographic performance in terms of sensitivity, resolution and etch resistance, the ZEP520A has emerged to a working horse in state-of-the-art EBL. However, the polymer characteristics of ZEP520A have remained unchanged since its development although some investigations indicated that its lithographic performance could be improved by adaptions of polymeric weights and dispersities. ${ }^{11,12}$ Moreover, in recent years there have been supplying difficulties of this resist and its price has been in constant increase. That is the reason why several resist materials have emerged on the market as potential alternatives to ZEP520A. For instance, SML (EM Resist Ltd.) has etch and resolution characteristics similar to ZEP520A and allows the generation of high aspect ratio structures, but in terms of sensitivity it is more similar to PMMA. ${ }^{13}$ Another material that has been advertised as ZEP520A replacement is CSAR 62 (ALLRESIST GmbH), a chemically semi-amplified resist for positive tone EBL that consists of an photo acid generator in addition to the same copolymeric material as ZEP520A. It was shown to provide similar resolution, sensitivity, and etch resistance as ZEP520A but leads to undesired resist residuals after development. ${ }^{14}$ Finally, the resist material gL2000 (Gluon Labs) is nearly identical to ZEP520A and, expectedly, it exhibits similar lithographic characteristics. ${ }^{15}$ Additionally, chemically amplified resists and EUV resists are commonly used for high resolution EBL. ${ }^{16}$

Against this background, we think that the development of a novel positive tone resist material for electron beam lithography as an alternative to the EBL materials, being presently on the market, seems to be desirable. Within this work we have been aiming for high resolution, high sensitivity as well as considerable etch resistance. We anticipated that an organic copolymeric material consisting of an acrylic, chlorine-containing monomer (acrylate A) and an acrylic monomer bearing aromatic side-groups (acrylate B) would be reasonable to start with. Acrylate A and acrylate B were expected to introduce high sensitivity and high etch resistance into the resist material, respectively. The systematic variation of comonomer ratio and molecular weights of the copolymers would allow for the investigation of the correlation between the copolymer characteristics and the lithographic performance during EBL processing (sensitivity, resolution, etch resistance). The goal of this work is a deep insight into these relationships and, finally, the development of mr-PosEBR, a tailored, new positive tone resist material for state-of-the-art EBL. 


\section{EXPERIMENTAL}

For this study several random copolymers of acrylate A and acrylate B with varying compositions were synthesized via a free-radical polymerization procedure with azobisisobutyronitrile (AIBN, Fluka) as thermally decomposing initiator, according to literature procedures. ${ }^{17}$ A gel permeation chromatography (GPC) system PL-GPC 50 Plus from Polymer Laboratories equipped with a refractive index detector was used to determine molecular weights of the synthesized copolymers. Tetrahydrofuran (Fisher, GPC grade) was used as the eluent. With a flow rate of $1.0 \mathrm{~mL} / \mathrm{min}$, samples were analyzed as polymer solutions with a concentration of ca. $1-2 \mathrm{mg} / \mathrm{mL}$. Polystyrene standards were used for instrument calibration and the determination of relative molecular weights. The composition of the copolymeric materials, that is the comonomer content, was estimated based on the refractive index of the materials which is known to be linearly dependent on the comonomer content in copolymers. ${ }^{18}$ A Metricon 2010/M Prism Coupler and solid polymer layers on silicon wafers were used to obtain the refractive indices of the copolymer materials. For the preparation of the resist solutions, the solid copolymer materials were dissolved in anisole (10\% by weight) and the obtained solutions were filtered through appropriate syringe filters (PTFE) with pore sizes of $0.1 \mu \mathrm{m}$. The resist solutions were spin-coated onto 4 “" silicon wafers $(4000 \mathrm{rpm}, 30 \mathrm{~s})$, which were subsequently baked on a hotplate $\left(150{ }^{\circ} \mathrm{C}, 1 \mathrm{~min}\right)$. The obtained uniform film thicknesses for the different resist formulations ranged from 300 to $400 \mathrm{~nm}$ as determined with a tabletop reflectometer FTPadv from Sentech. Exposure by electron beam lithography was performed with a RAITH150 Two Gaussian electron beam system (acceleration voltage $30 \mathrm{kV}$; beam current $38 \mathrm{pA}$; aperture diameter $10 \mu \mathrm{m}$; beam size: 1 $\mathrm{keV}$ using the $30 \mu \mathrm{m}$ aperture: $4 \mathrm{~nm}, 20 \mathrm{keV}$ using the $30 \mu \mathrm{m}$ aperture: $2 \mathrm{~nm}$ ) and a Vistec EBPG 5000+ electron beam system (acceleration voltage $100 \mathrm{kV}$, beam current $2 \mathrm{nA}$; aperture diameter $400 \mu \mathrm{m}$ ). After electron beam exposure, the substrates were manually developed in a bath of amyl acetate $(100 \%)$ at a constant clean room temperature of $21{ }^{\circ} \mathrm{C}$ for 30-60 s. Afterwards the sample was rinsed with isopropanol for $30 \mathrm{~s}$ and blown dry with nitrogen. The same process was applied to the electron beam resists ZEP520A (Zeon Corp.) and PMMA 495k (Microchem Corp.) for comparative investigations.

Resist contrast measurements were carried out by writing a contrast pattern consisting of a series of $60 \mu \mathrm{m}$ by $60 \mu \mathrm{m}(30$ $\mathrm{kV})$ or $200 \mu \mathrm{m}$ by $200 \mu \mathrm{m}(100 \mathrm{kV})$ rectangles at a distance of $40 \mu \mathrm{m}(30 \mathrm{kV})$ or $200 \mu \mathrm{m}(100 \mathrm{kV})$. This size was chosen to ensure that the proximity effect could be neglected at the center of the rectangles. The exposure dose for the different copolymers was incremented in various ranges for the different materials as shown in Table 1. In case of the contrast measurements at $30 \mathrm{kV}$, the samples were coated with $\mathrm{Al}$ metal $(50 \mathrm{~nm})$ by electron beam evaporation and the residual film thicknesses in the corresponding rectangles were determined by means of white light interferometry (a noncommercial white-light interferometer based on a Mireau interferometer was used). In case of the contrast measurements at $100 \mathrm{kV}$, film thicknesses were measured with a DEKTAK profilometer. For comparison, the initial film thicknesses were normalized to 1 and the normalized remaining film thicknesses were plotted versus the $\log (\operatorname{dose})$ values to obtain contrast curves. The contrast values were obtained by linear fits to the linear regions of the dose curves. Dose-to-clear values were calculated from the corresponding fitted lines. Typical exposure dose ranges were between $0-400 \mu \mathrm{C} / \mathrm{cm}^{2}$ (Table 1).

Table 1. Dose ranges, increments, and film thicknesses used in contrast measurements at $30 \mathrm{kV}$ (* for $100 \mathrm{kV}$ ).

\begin{tabular}{|l|l|l|l|}
\hline Resist material & Dose range $\left[\boldsymbol{\mu C} / \mathbf{c m}^{2}\right]$ & Increments $\left[\boldsymbol{\mu C} / \mathbf{c m}^{2}\right]$ & Initial film thickness $[\mathbf{n m}]$ \\
\hline Copolymer 1 & $16.4-400$ & 16.4 & 400 \\
\hline Copolymer 2 & $12.5-300$ & 12.5 & 320 \\
\hline Copolymer 3 & $20.8-500$ & 20.8 & 340 \\
\hline Copolymer 4 & $8.2-200$ & 8.2 & 360 \\
\hline Copolymer 5 & $2.5-100(20-345)^{*}$ & $2.5(5)^{*}$ & $320(420)^{*}$ \\
\hline Copolymer 6 & $7.5-300$ & 7.5 & 330 \\
\hline Copolymer 7 & $5-200$ & 5 & 340 \\
\hline ZEP520A & $8.2-200$ & 8.2 & 311 \\
\hline PMMA 495k & $7.5-300$ & 7.5 & 320 \\
\hline
\end{tabular}

For resolution investigations, different test patterns were written into the resist layers in order to estimate the possible resolution of the synthesized copolymers. After development, the resist structures were investigated by means of 
scanning electron microscopy (SEM, RAITH150 Two system, Zeiss Gemini column). Line edge roughness (LER), line width roughness (LWR) and critical dimension (CD) analyses were performed with selected scanning electron microscopy (SEM) pictures of resist patterns and patterns etched into silicon substrate using the software package SuMMIT (EUV Tech Inc.).

In order to investigate the pattern transfer by lift-off process, dense and sparse line-space patterns with gradually increasing line width were exposed. Developed samples were coated subsequently with $5 \mathrm{~nm}$ of titanium and $20 \mathrm{~nm}$ of gold using an electron gun evaporator at the rate of $1 \AA / \mathrm{s}$. Excessive metal film was lifted off with acetone via sonication and rinsed in isopropanol. The obtained metal patterns were inspected by SEM.

The dry etch resistance of the different new resist materials was evaluated by applying a fluorine based reactive-ion etching (RIE) process. Si wafers with patterned resist were etched using a Plasmalab System 100 (ICP180 source) from Oxford Instruments with the process gases $\mathrm{SF}_{6}(5 \mathrm{sccm})$ and $\mathrm{CF}_{4}(45 \mathrm{sccm})$, plasma power RIE $50 \mathrm{~W}$ and ICP $200 \mathrm{~W}$ $(\mathrm{DC}$ bias $330 \mathrm{~V})$, pressure $15 \mathrm{mTorr}$, table temperature $18^{\circ} \mathrm{C}$, for $30 \mathrm{~s}$. Etch depths before and after etching, including the remaining resist, were measured using a profilometer (KLA Tencor alpha step). Then, the resist was stripped with acetone (5 min). Finally, the depth was measured again to obtain the silicon etch depth. From these measurements, both the resist and silicon etch rates could be determined. In order to get a cross sectional view of the etched structures on silicon substrate, the wafer was cut by means of Focused Ion Beam (FIB Zeiss NVision 40).

The electron beam grayscale patterning was done with a $100 \mathrm{kV}$ system (Vistec EBPG 5000+) on 480 to $520 \mathrm{~nm}$ thick resist films. The resist was spun on silicon substrates that were oxygen plasma cleaned and then baked $\left(250^{\circ} \mathrm{C}, 5 \mathrm{~min}\right)$, previously. Post-apply baking of $1 \mathrm{~min}$ at $150^{\circ} \mathrm{C}$ was used as within the other investigations. The higher the exposure dose, the smaller is the molecular weight and thus the higher is the development rate: In a highly exposed resist region more resist is removed in the same period of time during development compared to a less exposed region. This allows to creating stepped or so called grayscale profiles. By using a contrast-curve-based approach, the dose required to achieve a certain structure height was applied in the desired region. The dose assignment and proximity calculation was done with a commercial software package (Layout BEAMER, GenISys GmbH Germany). The wet development was done in amyl acetate at $20^{\circ} \mathrm{C}$ and the samples were immediately rinsed in DI-water to stop the development and finally blown dry by nitrogen. The development was stopped once the regions to be cleared were free of resist. This ensured the correct height of all steps and avoided an overdevelopment.

Due to the chain scission upon electron exposure, the molecular weight as well as the glass transition temperature is reduced. ${ }^{19}$ The higher the exposure dose, the lower is the glass transition temperature. As a consequence, the viscosity of the resist during thermal reflow of a section with a higher dose is smaller compared to a section with a lower dose. Lower viscosities are equal to larger molecular mobilities. Thus, the higher the exposure dose is the faster is the reflow of these sections. The thermal reflow was conducted using a temperature controlled hotplate at $125^{\circ} \mathrm{C}$ for different periods of time. The glass transition temperature of the unexposed material is $125 \pm 4{ }^{\circ} \mathrm{C}$. After the reflow, the samples were immediately cooled to room temperature to "freeze" the reflow shape for further inspection. Crystal oriented cleaving and scanning electron microscopy inspection of the obtained cross-sections was used to get the reflowed profile. Elongated patterns of single lines, double-steps and multiple-steps were investigated. Further details on the sample preparation and the reflow behavior can be found in previous literature. ${ }^{20,21}$

\section{RESULTS AND DISCUSSION}

\subsection{Material synthesis and contrast measurements}

A series of acrylic copolymeric materials, that cover a range of compositions in terms of comonomer content (mass fractions of acrylate A and B), were synthesized by a common free-radical polymerization synthesis route from mixtures of the two monomers. ${ }^{17}$ The targeted molecular weights and comonomer ratios in the final copolymer products were achieved by the specific adjustment of the initiator concentration and the ratios of the comonomers $\mathrm{A}$ and $\mathrm{B}$ in the feed stock solutions, respectively. Within this study, seven different copolymer materials with mass fractions of acrylate A ranging from 37 to 63 weight percent were synthesized as shown in Table 2 (copolymers 1-7). Subsequently, coatings of the copolymers 1-7 on silicon wafers were subjected to contrast measurements at acceleration voltages of $30 \mathrm{kV}$ (Figure 2). 
Table 2. Molecular weight distributions of the synthesized copolymers and the sensitivity and contrast data obtained at an acceleration voltage of $30 \mathrm{kV} . M_{n}$ : number average molecular mass, $M_{w}$ : mass average molecular mass, PDI: polydispersity index; ${ }^{x}$ in the contrast measurement performed, reliable contrast values could not be obtained due to a strong scattering of the values (compare to Figure 2).

\begin{tabular}{|c|c|c|c|c|c|c|}
\hline \multirow[t]{2}{*}{ Resist material } & \multicolumn{2}{|c|}{ Molecular weight $[\mathrm{g} / \mathrm{mol}]$} & \multirow[t]{2}{*}{ PDI } & \multirow{2}{*}{$\begin{array}{l}\text { Mass fraction of } \\
\text { acrylate A [wt. \%] }\end{array}$} & \multirow{2}{*}{$\begin{array}{l}\text { Dose-to-clear } \\
{\left[\mu \mathrm{C} / \mathrm{cm}^{2}\right]}\end{array}$} & \multirow[t]{2}{*}{ Contrast $\gamma$} \\
\hline & $M_{n}$ & $M_{w}$ & & & & \\
\hline Copolymer 1 & 90500 & 160800 & 1.78 & 52 & 306 & 4.0 \\
\hline Copolymer 2 & 57400 & 94500 & 1.65 & 49 & 217 & 4.0 \\
\hline Copolymer 3 & 59200 & 92900 & 1.57 & 63 & $>450$ & 3.7 \\
\hline Copolymer 4 & 78500 & 137700 & 1.75 & 44 & 90 & 3.6 \\
\hline Copolymer 5 & 80900 & 134200 & 1.66 & 40 & 74 & 3.9 \\
\hline $\begin{array}{l}\text { Copolymer } 5 \\
(100 \mathrm{kV})\end{array}$ & 80900 & 134200 & 1.66 & 40 & 340 & 3.0 \\
\hline Copolymer 6 & 84700 & 136100 & 1.61 & 46 & 109 & 3.2 \\
\hline Copolymer 7 & 32900 & 66200 & 2.01 & 37 & 124 & 2.8 \\
\hline ZEP520A & $30500^{11}$ & $67000^{11}$ & $2.20^{11}$ & 50 & $>60$ & $--^{x}$ \\
\hline PMMA 495k & -- & 495000 & -- & -- & 146 & 6.1 \\
\hline
\end{tabular}

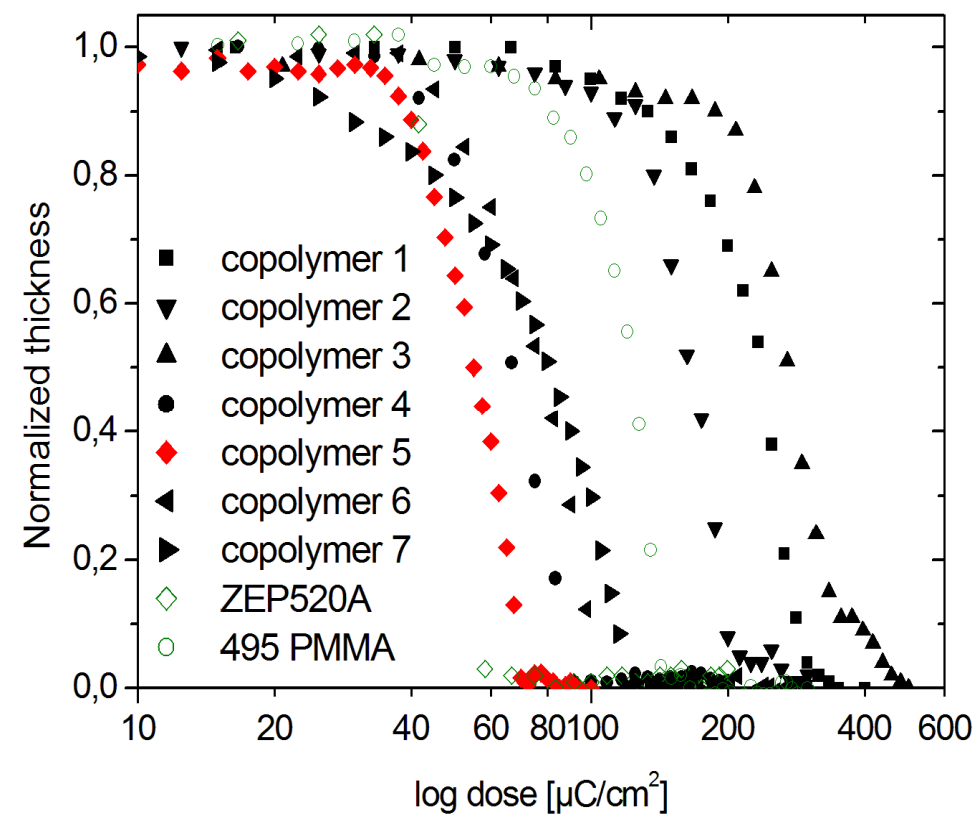

Figure 2. Contrast curves of the copolymers 1-7, the resist ZEP520A, and PMMA 495k, obtained at $30 \mathrm{kV}$ acceleration voltage; film thicknesses were between 300 and $400 \mathrm{~nm}$ (Table 1).

As can be seen from Figure 2, the dose-to-clear values, that is the sensitivities of the seven potential resist materials, are widely differing and range from values of $74 \mu \mathrm{C} / \mathrm{cm}^{2}$ for copolymer 5 to more than $450 \mu \mathrm{C} / \mathrm{cm}^{2}$ for copolymer 3 . At the same time, the contrast values do not exhibit such a large variation and adopt values between 2.8 and 4.0 (see also Table 2). The huge differences of the dose-to-clear values of the seven potential resist materials may be ascribed to their copolymer composition: The correlation between sensitivity of the copolymers and their composition is depicted more clearly in Figure 3 a. 

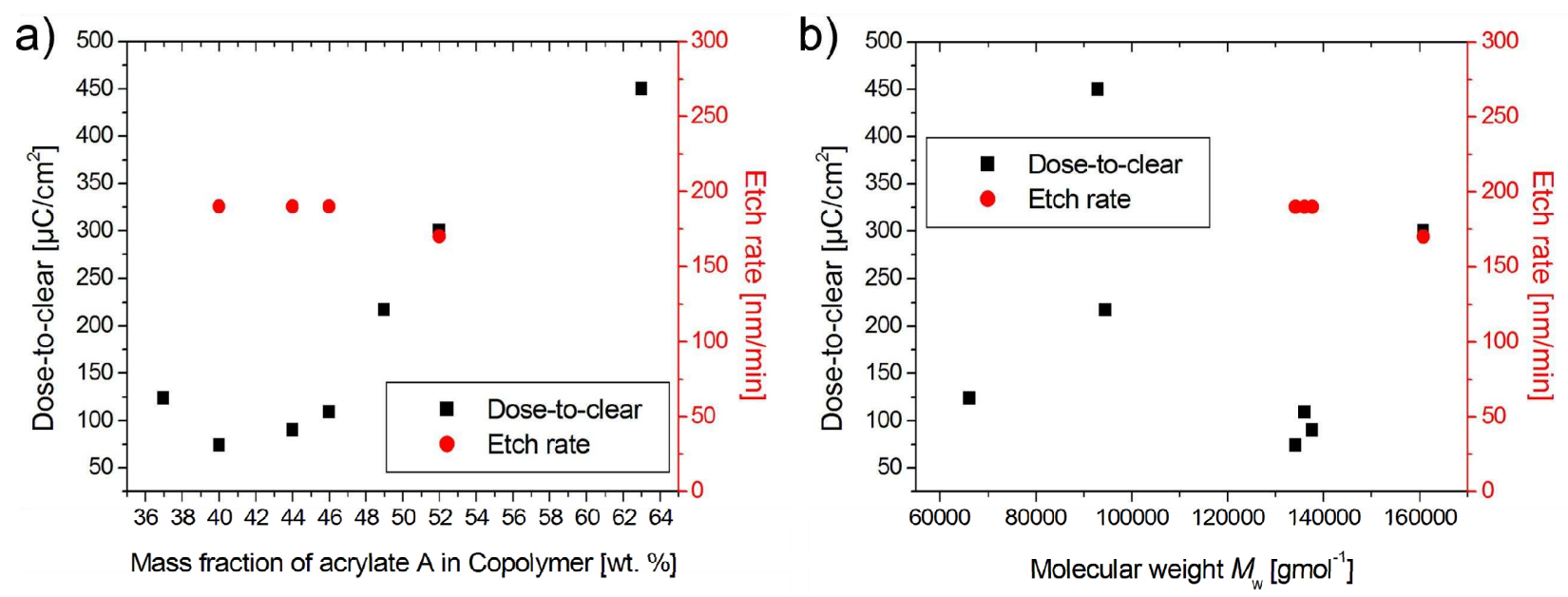

Figure 3. a) Plot of dose-to-clear values and etch rates vs. the mass fractions of comonomer A in the copolymer materials; b) Plot of dose-to-clear values and etch rates vs. molecular weights of the investigated copolymer materials. All sensitivity data were obtained with $30 \mathrm{kV}$ EBL.

Within the composition range investigated in this study, the lowest dose-to-clear values could be observed at mass fractions of acrylate A of around $40 \mathrm{wt}$. \%. Materials with this composition (copolymer 5) exhibit a dose-to-clear value of $74 \mu \mathrm{C} / \mathrm{cm}^{2}$. Consequently, copolymers with lower (copolymer 7) or higher (copolymers 1-4, 6) mass fractions of acrylate A show higher dose-to-clear values. Raising the mass fraction of acrylate $\mathrm{A}$ in the copolymeric materials above $40 \mathrm{wt}$ \% leads to the constant increase of the corresponding dose-to-clear values, so that, for example, the dose-to-clear value amounts to more than $450 \mu \mathrm{C} / \mathrm{cm}^{2}$ for copolymer 3 with $63 \mathrm{wt} . \%$ of acrylate A. This trend is in general agreement with a literature report on the positive tone electron beam resist behavior of a random copolymer of methyl $\alpha$ chloroacrylate and methacrylonitrile. In that study, the increasing incorporation of methyl $\alpha$-chloroacrylate lead to decreased electron beam sensitivity due to an increased susceptibility toward crosslinking. ${ }^{6}$

In contrast to the copolymer composition, molecular weight is known to have a much smaller effect on the sensitivity of positive tone materials than compared to negative tone electron beam resists. ${ }^{22}$ However, previous investigations with ZEP520A-like resist materials have shown that molecular weight and polymer dispersity can actually influence lithographic parameters such as sensitivity, line edge roughness (LER), and surface roughness. ${ }^{11,12}$ In Figure $3 \mathrm{~b}$ ) the dose-to-clear values of the seven copolymers were plotted against their molecular weights. Apparently, the sensitivities do not seem to be influenced significantly by the polymer masses, at least not in the range investigated. Hence, if there is an influence of molecular weight on the resist sensitivity, it is probably outperformed by the effect of the copolymer composition.

In comparison to the materials investigated herein, the well-known positive tone EBL resist ZEP520A, which is a 1:1 copolymer of methyl $\alpha$-chloroacrylate and $\alpha$-methylstyrene, ${ }^{8}$ shows a dose-to-clear value of around $60 \mu \mathrm{C} / \mathrm{cm}^{2}$ under identical process conditions. This means that the most sensitive material from this study, copolymer 5 , is comparably sensitive towards electron beams as the established material ZEP520A. Moreover, copolymer 5 is roughly twice as sensitive as PMMA (PMMA 495k, Microchem Corp.) exhibiting a dose-to-clear value of $146 \mu \mathrm{C} / \mathrm{cm}^{2}$ under identical conditions, too.

Copolymer 5 (mr-PosEBR), the most sensitive material to electrons accelerated at $30 \mathrm{kV}$, was additionally investigated by means of EBL at $100 \mathrm{kV}$. Under these conditions, the dose-to-clear amounts to $340 \mu \mathrm{C} / \mathrm{cm}^{2}$ and the contrast value to 3.0 (Figure 4). 


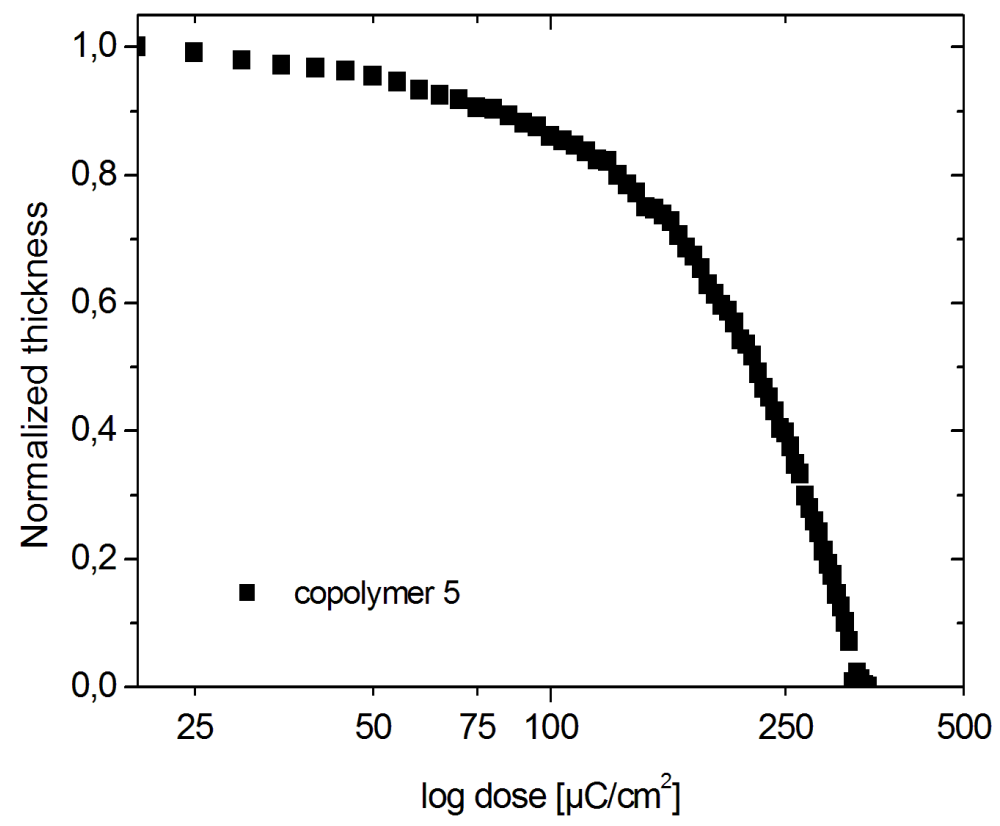

Figure 4. Contrast curve of copolymer 5; film thickness was $420 \mathrm{~nm}$ (see Table 1), acceleration voltage $100 \mathrm{kV}$.

\subsection{Resolution}

In addition to the contrast measurements described before, micro and nano patterns were written into layers of the copolymers 4 and 5 by means of electron beam lithography at $30 \mathrm{kV}$ as well as at $100 \mathrm{kV}$ in order to evaluate the materials' resolution capabilities. SEM pictures of this investigation are shown in Figure 5 and Figure 6.
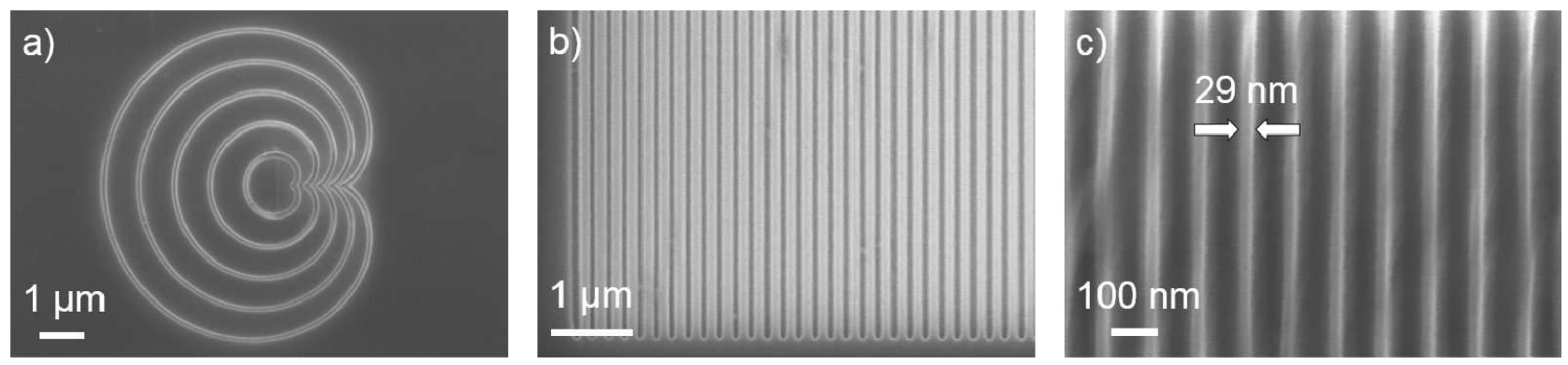

Figure 5. Resist patterns written into copolymer $4(30 \mathrm{kV})$ : a) cardioids structure, b) line and space pattern with a period of $200 \mathrm{~nm}$, and copolymer $5(30 \mathrm{kV})$ : c) grating pattern with $29 \mathrm{~nm}$ wide lines with a period of $100 \mathrm{~nm}$ (LER: $5.9 \mathrm{~nm}$, LWR: $16.8 \mathrm{~nm}$, CD: $29.3 \mathrm{~nm}$ ); initial film thicknesses were 360 (copolymer 4) and $320 \mathrm{~nm}$ (copolymer 5).

In Figure 5 a) a cardioids structure with a diameter of approximately $7 \mu \mathrm{m}$ is depicted (copolymer 4 ). The structure is well resolved and the $150 \mathrm{~nm}$ wide trenches are well developed. The same is true for the lines and spaces pattern with a period of $200 \mathrm{~nm}$ shown in Figure $5 \mathrm{~b}$ ). Due to the good contrast, the exposed areas are well developed while the unexposed areas remain unaffected. Even smaller patterns could be generated: In copolymer 5 - the most sensitive material in the previous contrast measurements - a grating pattern with a period of $100 \mathrm{~nm}$ was written very smoothly. Using adapted doses allowed the production of very thin lines of only $29 \mathrm{~nm}$ width.

Furthermore, Figure 6 a) shows a chirped grating pattern written into copolymer 5 with an acceleration voltage of 100 $\mathrm{kV}$ (initial layer thickness was $100 \mathrm{~nm}$, structures are coated with vaporized chromium of approximately $11 \mathrm{~nm}$ thickness). Dense lines with a pitch better than $50 \mathrm{~nm}$ were resolved, confirming the high resolution capabilities of copolymer 5. Moreover, lines and spaces patterns in $100 \mathrm{~nm}$ thick copolymer 5 were used for pattern transfer via lift-off and the resulting Ti/Au lines with the width of $35 \mathrm{~nm}$ are shown in Figure $6 \mathrm{~b}$ ). 


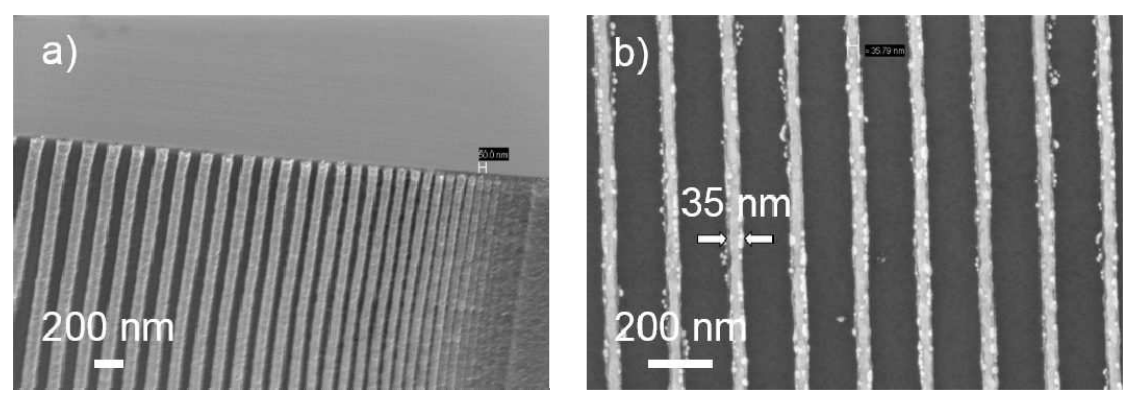

Figure 6. a) Chirped grating pattern written into copolymer $5(100 \mathrm{kV})$; b) Ti/Au lines via lift off from a resist pattern in a $100 \mathrm{~nm}$ thick layer of copolymer 5 (100 kV, LER: $12.4 \mathrm{~nm}$, LWR: $16.8 \mathrm{~nm}, \mathrm{CD}: 43.9 \mathrm{~nm})$.

Consequently, these results show that high resolution nano patterning of resist layers of the copolymers 4 and 5 is possible. In future, we will look more closely into the variation of development conditions as those are known to have a significant impact on the resolution, too. ${ }^{23}$

\subsection{Etch experiments on silicon}

With the copolymers $1,4,5$, and 6 dry etching experiments were performed. A RIE process with $\mathrm{CF}_{4}$ and $\mathrm{SF}_{6}$ as etch gases was applied to samples of the copolymer materials on silicon substrates and the resulting etch rates for the different copolymers are listed in Table 3. All etch rates are in the range between 170 and $190 \mathrm{~nm} / \mathrm{min}$ and are only slightly higher than the etch rate of ZEP520A $(150 \mathrm{~nm} / \mathrm{min})$ and significantly lower than the etch rate of silicon $(440 \mathrm{~nm} / \mathrm{min})$. Although there may be seen a tendency of lower etch rates for copolymer compositions with higher MCA contents and higher molecular weights, respectively, more data is required to confirm this assumption (see also Figure 3 a) and b)).

Table 3. Molecular weights of the investigated copolymers together with etch rates obtained in a RIE process $\left(\mathrm{CF}_{4} / \mathrm{SF}_{6}\right)$. Under identical process conditions the etch rates for silicon were $440 \mathrm{~nm} / \mathrm{min} ; M_{n}$ : mass average molecular mass.

\begin{tabular}{|c|c|c|c|c|}
\hline Resist material & $\begin{array}{l}\text { Molecular weight } \\
M_{w}[\mathrm{~g} / \mathrm{mol}]\end{array}$ & $\begin{array}{l}\text { Mass fraction of } \\
\text { acrylate A [wt. \%] }\end{array}$ & $\begin{array}{l}\text { Etch rate } \\
{[\mathrm{nm} / \mathrm{min}]}\end{array}$ & Etch selectivity to $\mathrm{Si}$ \\
\hline Copolymer 1 & 160800 & 52 & 170 & 2.6 \\
\hline Copolymer 4 & 137700 & 44 & 190 & 2.3 \\
\hline Copolymer 5 & 134200 & 40 & 190 & 2.3 \\
\hline Copolymer 6 & 136100 & 46 & 190 & 2.3 \\
\hline ZEP520A & $67000^{11}$ & 50 & 150 & 2.9 \\
\hline
\end{tabular}

In order to probe the pattern transfer capabilities of the new resist material mr-PosEBR (copolymer 5), micro- and nanostructures written into mr-PosEBR (copolymer 5) were used as etch masks in the above mentioned RIE process. In Figure 7 SEM pictures of the resulting structures etched into silicon are shown. These demonstrate that with the present etch process micro and nano structures in layers of mr-PosEBR (copolymer 5) can be transferred into the underlying silicon substrate with high fidelity. In order to obtain a more detailed impression of the etched structures the silicon wafer was cut by means of focused ion beams and the corresponding cross section was investigated by SEM. In Figure 7 e) a lines and spaces pattern with a period of $200 \mathrm{~nm}$ in silicon is shown. Again, the high fidelity of the pattern transfer can be confirmed. However, a slight tapering is observed at the front face of the lines. This is due to a non-perfectly optimized dry etch process and we are confident that further optimization of the etch conditions will lead to vertical sidewalls. In conclusion, mr-PosEBR (copolymer 5) as well as the other copolymers investigated in this work show promising etch behavior and future work will investigate their stabilities and pattern transfer capabilities under different etching conditions (chlorine plasma, Ar sputtering, etc.). 

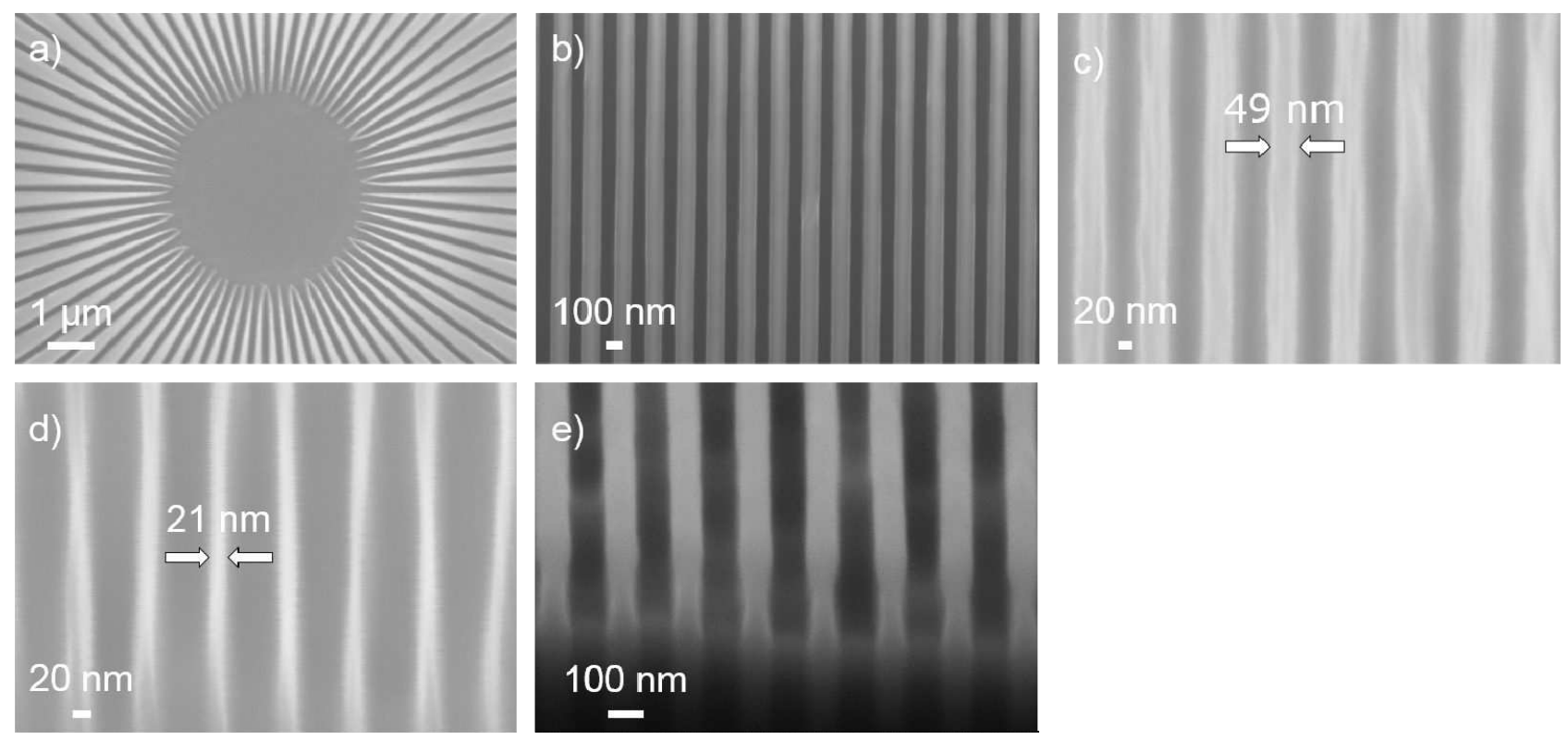

Figure 7. SEM pictures of micro and nano patterns of different dimension in silicon, etched with mr-PosEBR (copolymer 5) as the mask (resist structures generated at $30 \mathrm{kV}$ ): a) Siemens star structure, b) line and space pattern with a period of $200 \mathrm{~nm}$ (LER: $5.2 \mathrm{~nm}$, LWR: $10.5 \mathrm{~nm}$, CD: $110 \mathrm{~nm}$ ); c) line and space pattern with a period of $100 \mathrm{~nm}$ (LER: $3.7 \mathrm{~nm}$, LWR: $4.9 \mathrm{~nm}, \mathrm{CD}: 49 \mathrm{~nm}$ ); d) grating pattern with $21 \mathrm{~nm}$ wide lines with a period of $80 \mathrm{~nm}$; e) cross-sectional view of a line and space pattern (period of the lines is $200 \mathrm{~nm}$ ).

\subsection{Grayscale patterning and thermal reflow performance}

Basically, the doses required to get a certain relative height can be read from Figure 4. Using the mentioned software approach, the doses were assigned taking even the proximity effect into account. As a consequence of the controlled development, the step profiles were obtained as designed. This demonstrated the good usability of mr-PosEBR for grayscale patterning. Overall, the grayscale patterning performance was found to be very similar to PMMA $120 \mathrm{k}^{20}$ and ZEP520A. ${ }^{24}$ Figure 8 gives some examples of high resolution multilevel patterns. Step widths down to $240 \mathrm{~nm}$ in a 480 $\mathrm{nm}$ thick film of mr-PosEBR were realized with good fidelity (Figure 8c) and e)). Also five level configurations were realized with a clear separation of each step (Figure 8d) and e)). As a rule of thumb, steps being smaller than $50 \%$ of the resist height (Figure 8c) and e)) will start to transform into a continuous step profile due to lateral development. A typical effect of the grayscale patterning using a single exposure and a single development, as it is done in this work, is the increasing inclination of the step-to-step sidewall. ${ }^{24}$ This is clearly visible in Figure 8c) and e): The deeper the levels are buried in the initial resist film, the more inclined is the step-to-step sidewall. The origin of this inclination is a lateral removal of the exposed steps during the isotropic development process. The smaller the step width is the more does the lateral development influence the shape of the exposed step. 

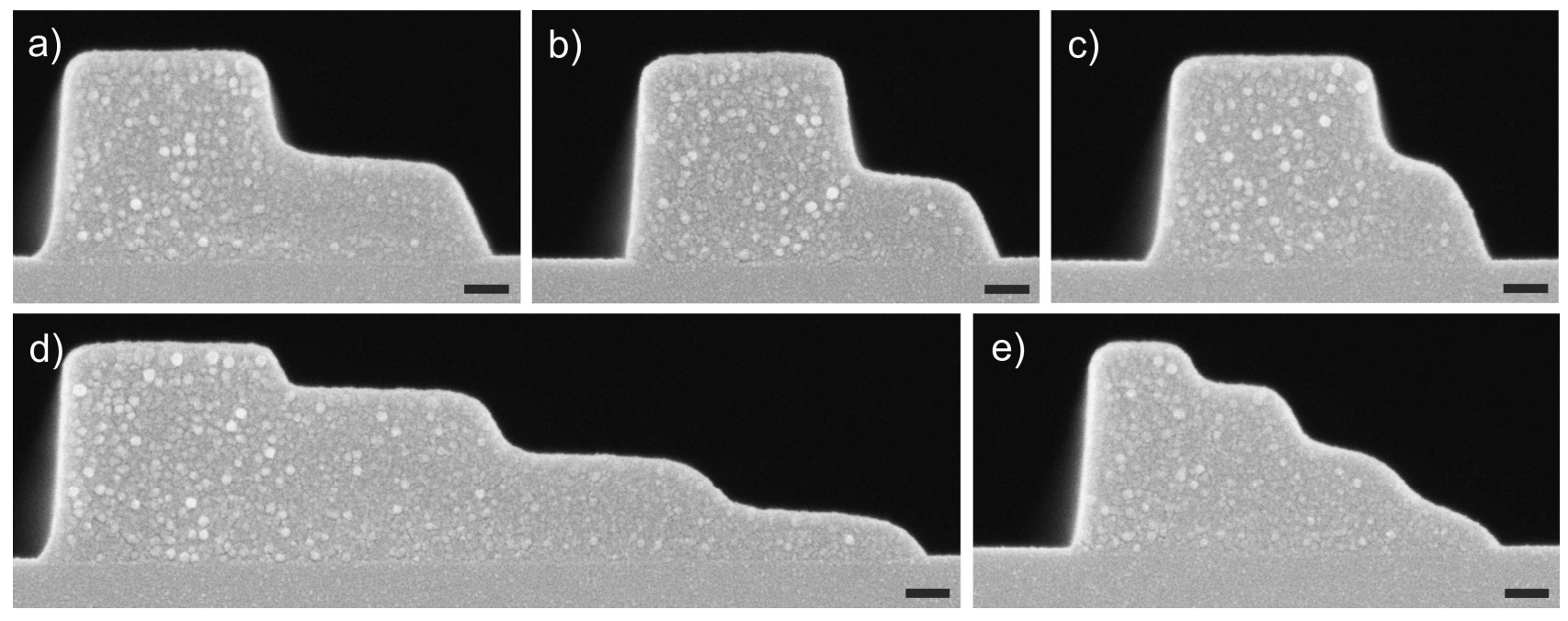

Figure 8 . Overview on grayscale patterning performance in a 480-520 nm thick mr-PosEBR film: double-step pattern (100 $\%$ and $50 \%$ original height) having a) $480 \mathrm{~nm}$ wide exposed and unexposed section, b) $480 \mathrm{~nm}$ wide unexposed and $360 \mathrm{~nm}$ wide exposed section, c) $480 \mathrm{~nm}$ wide unexposed and $240 \mathrm{~nm}$ wide exposed section as well as multi-step pattern of one unexposed and three exposed sections (100\%, $75 \%, 50 \%, 25 \%$ original height) having d) $480 \mathrm{~nm}$ wide steps and e) $240 \mathrm{~nm}$ wide steps (all scale bars $100 \mathrm{~nm}$ ).

Following the grayscale patterning, an even higher feature variety including convex and concave features can be achieved by reflow of the grayscale patterns at a defined temperature. This process is termed thermally activated selective topography equilibration (TASTE) ${ }^{19}$ and uses the before mentioned differences in the glass transition temperature of differently exposed sections. By choosing a reflow temperature of $125^{\circ}$, the glass transition temperature of unexposed mr-PosEBR, a very high selectivity between exposed and unexposed sections was achieved. While unexposed sections (Figure 9a) remained stable during reflow, exposed section (Figure 9c) reflowed very fast and approached a constant curvature feature as it is typical for an unconstrained surface-energy driven optimization process. This indicated a much higher mobility of the exposed section and confirms the assumed molecular weight reduction upon electron beam exposure. Depending on the aspect ratio, also unexposed features can collapse during reflow (Figure $9 \mathrm{~b})$. This is mainly due to the relative amount of material that moves even at this temperature very slowly along the substrate: While this small amount of material being displaced does not change the overall shape in Figure 9a, it hast a significant impact on the shape in Figure 9c. By combining unexposed and exposed sections into a more complex configuration (Figure 9d and e) asymmetric features due to a stabilizing part and a selectively reflowing part can be achieved. Such features are of high interest for micro-optical devices and can be produced in a wide range of resist thicknesses ranging from a few microns down to some $10 \mathrm{~nm}$. 


\section{a) wide single line remaining stable}
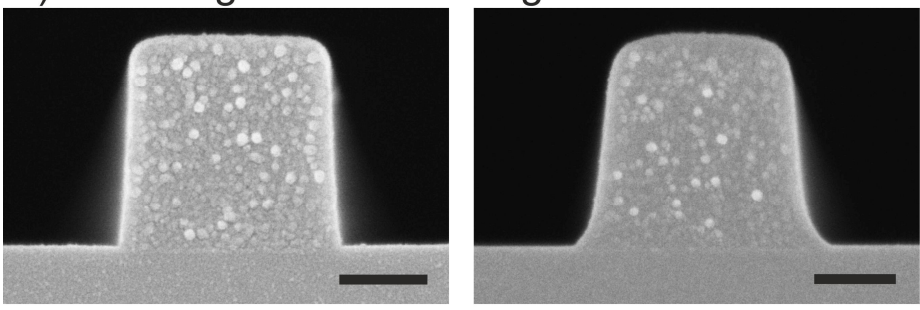

b) small single line collapsing

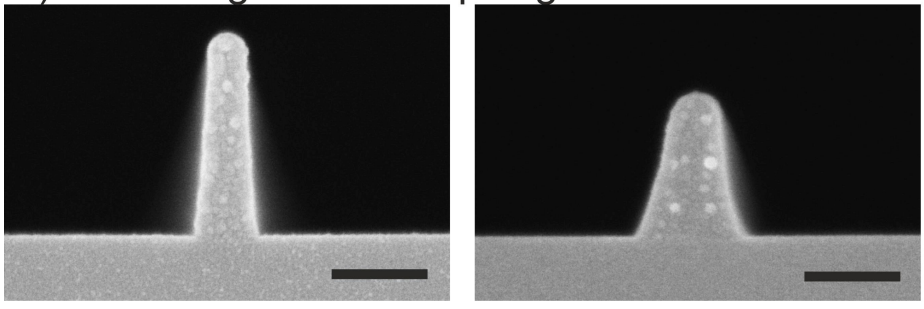

c) wide single line approaching constant curvature

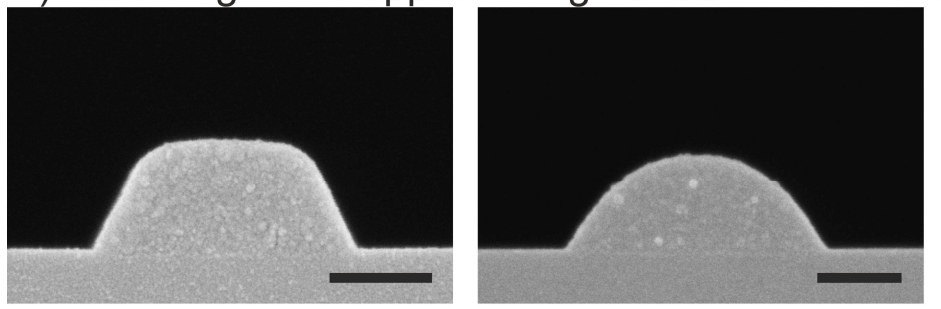

\section{d) double step transformed into asymmetric feature}
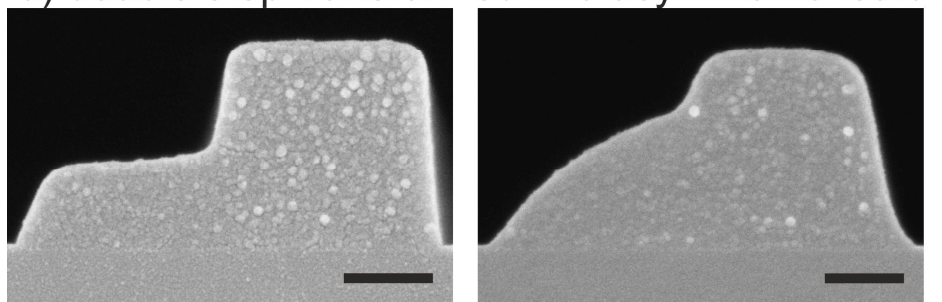

e) multi-step transformed into micro-prism

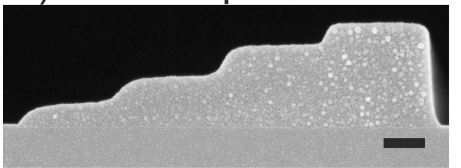

as developed

(initial geometry)

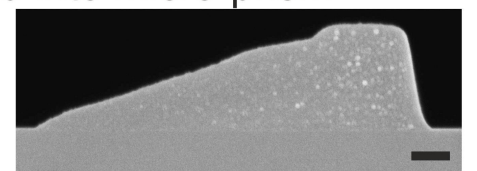

20 min @ $125^{\circ} \mathrm{C}$

$\left(\mathrm{T}_{\mathrm{g}} \sim 125^{\circ} \mathrm{C}\right)$

Figure 9. Comparison of grayscale features after development and as they appear after reflow at $125^{\circ} \mathrm{C}$ for 20 min: a) 480 $\mathrm{nm}$ wide unexposed and isolated line that remains stable during reflow while b) a $100 \mathrm{~nm}$ wide unexposed and isolated line collapses during reflow due to the higher aspect ratio, c) exposed single and isolated line of about $50 \%$ of the original resist height being transformed into a constant curvature shape upon reflow due to a reduced glass transition temperature and viscous material flow, d) a grayscale feature consisting of unexposed resist beside an exposed step with $50 \%$ of the original height undergoing a selective reflow and forming thus an asymmetric feature, e) a multistep pattern consisting of one unexposed step beside three exposed steps of $75 \%, 50 \%$ and $25 \%$ of the original resist height being transformed into a micro-prism due to selective reflow; all scale bars $200 \mathrm{~nm}$. 


\section{CONCLUSIONS}

In this contribution the development and lithographic evaluation of a novel positive tone resist material, mr-PosEBR, for high resolution electron beam lithography is described. By varying the copolymeric composition of the resist material, such as the comonomer mass fractions and the copolymer molecular weight, the lithographic sensitivity towards electron beams could be maximized. With doses-to-clear of below $100 \mu \mathrm{C} / \mathrm{cm}^{2}$ and contrast values of around 4 (30 $\mathrm{kV} \mathrm{EBL}$ ), the resist mr-PosEBR is approximately two times more sensitive than PMMA 495k and performs comparably to the known high resolution resist ZEP520A. The etch stability of mr-PosEBR in a fluorine based RIE process is significantly higher than the one of PMMA and similar to ZEP520A. Grating patterns with a period of $100 \mathrm{~nm}$ and a line width of $29 \mathrm{~nm}$ could be generated in uniform resist layers of mr-PosEBR with $30 \mathrm{kV}$ EBL and were subsequently transferred into the underlying silicon substrate by RIE. Further, the generation of $35 \mathrm{~nm}$ wide Ti/Au lines via lift-off corroborates the pattern transfer capabilities of mr-PosEBR. The usability of mr-PosEBR as potential resist for thermally activated selective topography equilibration (TASTE) was explored. It was shown that mr-PosEBR is well suited for grayscale electron beam lithography. The thermoplastic behavior of mr-PosEBR allowed for a selective thermal reflow of only the previously exposed sections resulting in smooth, continuously sloped structures. The possibility of generating various features in mr-PosEBR on the micro- and nanometer scale in combination with its considerable etch stability should enable the straightforward generation of 3D patterns in the underlying substrate. Consequently, mr-PosEBR represents an additional alternative resist material for high resolution positive tone electron beam lithography. In future, additional lithographic investigations will be performed that will add up to a comprehensive evaluation of this novel resist material mr-PosEBR.

\section{ACKNOWLEDGEMENT}

We thank Martin Messerschmidt and Arne Schleunitz for valuable scientific discussions.

\section{REFERENCES}

[1] Stepanova, M. and Dew, S. [Nanofabrication, Techniques and principles], Springer Vienna, Vienna, (2012).

[2] a) McCord, M. A. et al., "REBL: design progress toward $16 \mathrm{~nm}$ half-pitch maskless projection electron beam lithography," Proc. SPIE 8323, 832311 (2012). b) Klein, C., Loeschner, H. and Platzgummer, E., "50-keV electron multibeam mask writer for the 11-nm HP node: first results of the proof-of-concept electron multibeam mask exposure tool," J. Micro/Nanolith. MEMS MOEMS 11(3), 31402 (2012). c) Belledent, J. et al., "Sub20nm hybrid lithography using optical, pitch-division, and e-beam," Proc. SPIE 8323, 83230F (2012). d) Slot, E. et al., "MAPPER: high throughput maskless lithography," Proc. SPIE 6921, 69211P (2008).

[3] Grigorescu, A. E. and Hagen, C. W., "Resists for sub-20-nm electron beam lithography with a focus on HSQ: state of the art," Nanotechnology 20(29), 292001 (2009).

[4] Hatzakis, M., "Electron Resists for Microcircuit and Mask Production," J. Electrochem. Soc. 116(7), 1033-1037 (1969).

[5] Lai, J. H., Shepherd, L. T., Ulmer, R. and Griep, C., "Studies of methyl methacrylate-methyl $\alpha$-chloroacrylate, copolymers and poly(methyl $\alpha$-chloroactylate) as electron sensitive positive resists," Polymer Engineering \& Science 17(6), 402-405 (1977).

[6] Lai, J. H., Helbert, J. N., Cook, C. F. and Pittman, C. U., "Positive electron-beam resist behavior for methacrylonitrile and methyl $\alpha$-chloroacrylate polymers and copolymers," J. Vac. Sci. Technol. 16(6), 19921995 (1979).

[7] Harada, K., Kogure, O. and Murase, K., "Poly (phenyl methacrylate-co-methacrylic acid) as a dry-etching durable positive electron resist," IEEE Trans. Electron Devices 29(4), 518-524 (1982). 
[8] Nishida, T., Notomi, M., Iga, R. and Tamamura, T., "Quantum Wire Fabrication by E-Beam Lithography Using High-Resolution and High-Sensitivity E-Beam Resist ZEP-520," Jpn. J. Appl. Phys. 31(Part 1, No. 12B), 45084514 (1992).

[9] Koshelev, K., Ali Mohammad, M., Fito, T., Westra, K. L., Dew, S. K. and Stepanova, M., "Comparison between ZEP and PMMA resists for nanoscale electron beam lithography experimentally and by numerical modeling," J. Vac. Sci. Technol. B 29(6), 06F306 (2011).

[10] Babu, G. N., Narula, A., Hsu, S. L. and Chien, J. C. W., "Radiolysis of resist polymers. 1. Poly(methyl $\alpha-$ haloacrylates) and copolymers with methyl methacrylate," Macromolecules 17(12), 2749-2755 (1984).

[11] Yamaguchi, T., Yamazaki, K. and Namatsu, H., "Influence of molecular weight of resist polymers on surface roughness and line-edge roughness," J. Vac. Sci. Technol. B 22(6), 2604 (2004).

[12] Otani, M., Asada, H., Tsunoda, H., Kunitake, M., Ishizaki, T., Miyagawa, R. and Konishi, T., "Improvement of polymer type EB resist sensitivity and line edge roughness," Proc. SPIE 8081, 808107 (2011).

[13] Gangnaik, A., Georgiev, Y. M., McCarthy, B., Petkov, N., Djara, V. and Holmes, J. D., "Characterisation of a novel electron beam lithography resist, SML and its comparison to PMMA and ZEP resists," Microelectron. Eng. 123, 126-130 (2014).

[14] Thoms, S. and Macintyre, D. S., "Investigation of CSAR 62, a new resist for electron beam lithography," J. Vac. Sci. Technol. B 32(6), 06FJ01 (2014).

[15] Hobbs, R. G., "Lithographic Evaluation of gL-2000: A High-Resolution Resist for Electron-Beam Lithography," presented at EIPBN, San Diego, California, USA, 29 May 2015.

[16] a) Tsubaki, H., Naulleau, P. P., Tarutani, S., Inoue, N., Takizawa, H. and Goto, T., "EUV resist materials design for 15nm half pitch and below," Proc. SPIE 8679, 867905 (2013). b) Yang, D. X., Frommhold, A., Xue, X., Palmer, R. E. and Robinson, A. P. G., "Chemically amplified phenolic fullerene electron beam resist", J. Mater. Chem. C 2(8), 1505 (2014).

[17] Odian, G. G. [Principles of polymerization], Wiley-Interscience, Hoboken, N.J, (2004).

[18] Kwon, J. Y., Kim, B. G., Do, J. Y., Ju, J.-J. and Park, S. K., "Polarizing group attached acrylates and polymers viewing high refractive index," Macromol. Res. 15(6), 533-540 (2007).

[19] Schleunitz, A., Guzenko, V. A., Messerschmidt, M., Atasoy, H., Kirchner, R. and Schift, H., "Novel 3D microand nanofabrication method using thermally activated selective topography equilibration (TASTE) of polymers," Nano Convergence 1(7), 1-8 (2014).

[20] Kirchner, R., Schleunitz, A. and Schift, H., "Energy-based thermal reflow simulation for 3D polymer shape prediction using Surface Evolver," J. Micromech. Microeng. 24(5), 55010 (2014).

[21] Kirchner, R. and Schift, H., "Mobility based 3D simulation of selective, viscoelastic polymer reflow using surface evolver," J. Vac. Sci. Technol. B 32(6), 06F701 (2014).

[22] Con, C., Dey, R., Ferguson, M., Zhang, J., Mansour, R., Yavuz, M. and Cui, B., "High molecular weight polystyrene as very sensitive electron beam resist," Microelectron. Eng. 98, 254-257 (2012).

[23] a) Yamaguchi, T. and Namatsu, H., "Effect of developer molecular size on roughness of dissolution front in electron-beam resist," J. Vac. Sci. Technol. B 22(3), 1037 (2004). b) Cord, B., Lutkenhaus, J. and Berggren, K. K., "Optimal temperature for development of poly(methylmethacrylate)," J. Vac. Sci. Technol. B 25(6), 2013 (2007). c) Shokouhi, B., Zhang, J. and Cui, B., "Very high sensitivity ZEP resist using MEK:MIBK developer," Micro Nano Lett. 6(12), 992 (2011).

[24] Kirchner, R., Guzenko, V. A., Vartiainen, I., Chidambaram, N. and Schift, H., "ZEP520A - a resist for electronbeam grayscale lithography and thermal reflow," Microelectron. Eng. (2016), doi: 10.1016/j.mee.2016.01.017. 